\title{
Stability of Switched Systems with Average Dwell-Time ${ }^{1}$
}

\author{
João P. Hespanha \\ hespanha@usc .edu \\ Electrical Engineering-Systems \\ University of Southern California
}

\author{
A. Stephen Morse \\ morse@sysc.eng. yale.edu \\ Dept. Electrical Engineering \\ Yale University
}

\begin{abstract}
It is shown that switching among stable linear systems results in a stable system provided that switching is "slow-on-the-average." In particular, it is proved that exponential stability is achieved when the number of switches in any finite interval grows linearly with the length of the interval, and the growth rate is sufficiently small. Moreover, the exponential stability is uniform over all switchings with the above property. For switched systems with inputs this guarantees that several input-to-state induced norms are bounded uniformly over all slow-on-the-average switchings. These results extend to classes of nonlinear switched systems that satisfy suitable uniformity assumptions. In this paper it is also shown that, in a supervisory control context, scale-independent hysteresis can produce switching that is slow-on-the-average and therefore the results mentioned above can be used to study the stability of hysteresis-based adaptive control systems.
\end{abstract}

\section{Introduction}

Consider a family of $n \times n$ stability matrices $\mathcal{A} \triangleq\left\{A_{p}\right.$ : $p \in \mathcal{P}\}$ indexed by the parameter $p$ taking values on a set $\mathcal{P}$. It is well known that, even if each matrix in $\mathcal{A}$ is asymptotically stable, the time-varying switched system

$$
\dot{x}=A_{\sigma} x
$$

may have unbounded solutions for some "switching signals" $\sigma$. Here, a switching signal is simply a piecewise constant signal taking values on the index set $\mathcal{P}$. However, it is also known that (1) is exponentially stable if the interval between any two consecutive discontinuities of $\sigma$ is sufficiently large.

Given a positive constant $\tau_{D}$, let $\mathcal{S}\left[\tau_{D}\right]$ denote the set of all switching signals with interval between consecutive discontinuities no smaller than $\tau_{D}$. The constant $\tau_{D}$

\footnotetext{
${ }^{1}$ This research was supported by ONR (grant N00014-97-10946), AFOSR, ARO, and NSF.
}

is called the (fixed) dwell-time. It was shown, e.g., in [1, Lemma 2], that one can pick $\tau_{D}$ sufficiently large so that (1) is exponentially stable for every $\sigma \in \mathcal{S}\left[\tau_{D}\right]$. In fact, there exist positive constants $c, \lambda$ such that

$$
\left\|\Phi_{\sigma}(t, \tau)\right\| \leq c e^{-\lambda(t-\tau)}, \quad \forall t \geq \tau \geq 0, \sigma \in \mathcal{S}\left[\tau_{D}\right],
$$

where $^{1} \Phi_{\sigma}(t, \tau)$ denotes the state transition matrix of (1). In this paper we show that a similar result still holds when $\mathcal{S}\left[\tau_{D}\right]$ is enlarged to contain signals that occasionally have consecutive discontinuities separated by less than $\tau_{D}$, but for which the average interval between consecutive discontinuities is no less than $\tau_{D}$. We proceed to formalize this concept of "average dwelltime". For each switching signal $\sigma$ and each $t \geq \tau \geq 0$, let $N_{\sigma}(t, \tau)$ denote the number of discontinuities of $\sigma$ in the open interval $(\tau, t)$. For given $N_{0}, \tau_{D}>0$, we denote by $\mathcal{S}_{\text {ave }}\left[\tau_{D}, N_{0}\right]$ the set of all switching signals for which

$$
N_{\sigma}(t, \tau) \leq N_{0}+\frac{t-\tau}{\tau_{D}} .
$$

The constant $\tau_{D}$ is called the average dwell-time and $N_{0}$ the chatter bound. Clearly, $\mathcal{S}\left[\tau_{D}\right] \subset \mathcal{S}_{\text {ave }}\left[\tau_{D}, 1\right]$. In this paper we show that, if $\tau_{D}$ is sufficiently large, a bound like (2) actually holds for every $\sigma \in \mathcal{S}_{\text {ave }}\left[\tau_{D}, N_{0}\right]$, with arbitrary $N_{0}$, and not only for the switching signals in $\mathcal{S}\left[\tau_{D}\right]$.

Systems like (1) arise in an adaptive context when a high-level, logic-based supervisor orchestrates the switching between a family of candidate controllers so as to achieve some desired behavior for the closed-loop system $[2,3,1,4,5,6,7,8,9,10,11,12,13]$. The need for switching usually arises from the fact that no single candidate controller would be capable, by itself, of guaranteeing stability and good performance when connected with a poorly modeled process. In several of these algorithms the supervisor guarantees, by construction, that there is a minimum time $\tau_{D}$ between consecutive switchings $[2,3,1,4,5,6,7]$. The dwelltime $\tau_{D}$ is then a design parameter ${ }^{2}$ chosen so that (1)

\footnotetext{
${ }^{1}$ Given a matrix $A$, we denote by $\|A\|$ the largest singular value of $A$.

${ }^{2}$ In $[1,5]$ the dwell-time is actually chosen arbitrarily and (1) is then "massaged" by output injection to make it exponentially stable for that particular dwell-time.
} 
is exponentially stable.

Dwell-time switching supervisors force every candidate controller to remain in the loop for, at least, $\tau_{D}$ units of time, thus guaranteeing a fixed dwell-time of $\tau_{D}$. Unfortunately, with nonlinear system this may lead to finite escape of the closed-loop. Adaptive switching algorithms for nonlinear system have therefore avoided a fixed dwell-time, and have been mostly ${ }^{3}$ based on hysteresis switching $[15,16]$, or on its more recent scaleindependent version $[9,17]$.

To date, the analysis of algorithms based on hysteresis switching relied heavily on showing that switching stops in finite time $[8,9,10,11,12,13]$. However, in the presence of noise and disturbance inputs, this is hardly the case. In fact, the only known switching algorithms for which switching can be proved to stop in finite time, even in the presence of noise/disturbances, are those for which an upper bound on these signals is known a priori, or effectively estimated online $[3,18]$. Unfortunately, even in the noiseless case, these algorithms usually lead to bad transient responses.

It turns out that, making use of the results in [17], one can show that, although scale-independent hysteresis switching does not guarantee the existence of a fixed dwell-time between switchings, it can produce switching that is slow-on-the-average. This allows us to analyze supervisory control switching algorithms based on scale-independent hysteresis, even in the presence of noise. To the best of our knowledge, this is the first time that an hysteresis-based switching algorithm is analyzed without relying on switching stopping.

This paper is organized as follows. In Section 2, we formalize the notion of exponential stability, uniform over a class of switching signals. We show that with this type of stability one can compute bounds on several induced input-to-state norms that are uniform over the same class of switching signals. Section 3 contains the main result of this paper, namely that, for linear systems, slow average dwell-time guarantees uniform exponential stability. In Section 4, this result is extended to certain classes of nonlinear systems. Section 5 makes use of previous results in the analysis of a supervisory control algorithm that uses scale-independent hysteresis switching logic. Finally, Section 6 contains some concluding remarks and directions for future research. The reader is referred to [19] for the proofs of some of the results presented here.

\section{Uniform Stability}

Consider again the switched system

$$
\dot{x}=A_{\sigma} x \text {. }
$$

\footnotetext{
${ }^{3}$ An exception is the supervisor based on dwell-time switching described in [14]. However, because of finite escape, the stability results given are only semi-global.
}

Given some family of piecewise constant switching signals $\mathcal{S}$, we say that (1) is uniformly exponentially stable over $\mathcal{S}$ if there exist positive constants $c$ and $\lambda$ such that, for each $\sigma \in \mathcal{S}$,

$$
\left\|\Phi_{\sigma}(t, \tau)\right\| \leq c e^{-\lambda(t-\tau)}, \quad \forall t \geq \tau \geq 0,
$$

where $\Phi_{\sigma}(t, \tau)$ is the state transition matrix of (1). When we want to emphasize the rate of decay in the above bound we add that (1) has stability margin $\lambda$.

Take now a bounded family of $n \times m$ matrices $\mathcal{B} \triangleq\left\{B_{p}\right.$ : $p \in \mathcal{P}\}$, also parameterized by the elements of the index set $\mathcal{P}$, and consider the $m$-input system

$$
\dot{z}=A_{\sigma} z+B_{\sigma} u
$$

where $u:[0, \infty) \rightarrow \mathbb{R}^{m}$ denotes some piecewise continuous input and $\sigma$ some switching signal in $\mathcal{S}$. We show next that uniform exponential stability of (1) over $\mathcal{S}$ implies that several induced norms of (3) are uniformly bounded over $\mathcal{S}$.

Given a nonnegative constant $\lambda$, we say that (3) has input-to-state $e^{\lambda t}$-weighted, $\mathcal{L}_{2}$-induced norm uniformly bounded over $\mathcal{S}$ if there exist finite constants $\mathfrak{g}$, $\mathfrak{g}_{0}$ such that, for each piecewise continuous input $u$ and each $\sigma \in \mathcal{S}$,

$$
\begin{aligned}
& \left(\int_{0}^{t} e^{2 \lambda \tau}\|z(\tau)\|^{2}\right)^{\frac{1}{2}} \leq \\
& \quad \mathfrak{g}\left(\int_{0}^{t} e^{2 \lambda \tau}\|u(\tau)\|^{2}\right)^{\frac{1}{2}}+\mathfrak{g}_{0}\|z(0)\|, \quad t \geq 0
\end{aligned}
$$

along solutions to (3) ${ }^{4}$. If (4) is replaced by

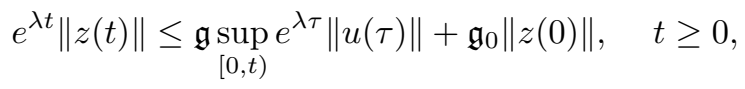

we say that (3) has input-to-state $e^{\lambda t}$-weighted, $\mathcal{L}_{\infty}$ induced norm uniformly bounded over $\mathcal{S}$, and if (4) is replaced by

$$
e^{\lambda t}\|z(t)\| \leq \mathfrak{g}\left(\int_{0}^{t} e^{2 \lambda \tau}\|u(\tau)\|^{2}\right)^{\frac{1}{2}}+\mathfrak{g}_{0}\|z(0)\|, \quad t \geq 0
$$

we say that $(3)$ has input-to-state $e^{\lambda t}$-weighted, $\mathcal{L}_{2}$ to- $\mathcal{L}_{\infty}$-induced norm uniformly bounded over $\mathcal{S}$. The finiteness of the three induced norms above is crucial to the analysis of adaptive switching algorithms, especially when noise and unmodeled dynamics are taken into account (cf. Section 5 and $[3,1,4,5,6,7,19]$ ). The following Lemma is proved in [19].

Lemma 1 Given a family $\mathcal{S}$ of piecewise constant switching signals, if (1) is uniformly exponentially stable over $\mathcal{S}$, with stability margin $\lambda_{0}$, then, for any $\lambda \in\left[0, \lambda_{0}\right),(3)$ has input-to-state $e^{\lambda t}$-weighted, $\mathcal{L}_{2}$ induced norm uniformly bounded over $\mathcal{S}$. Similarly for the $\mathcal{L}_{\infty}$ and $\mathcal{L}_{2}$-to- $\mathcal{L}_{\infty}$ induced norms.

\footnotetext{
${ }^{4}$ Given a vector $x$, we denote by $\|x\|$ the Euclidean norm of $x$
} 


\section{Average Dwell Time}

Let us recall that, for given $N_{0}, \tau_{D}>0, \mathcal{S}_{\text {ave }}\left[\tau_{D}, N_{0}\right]$ denotes the set of all switching signals with average dwell time $\tau_{D}$ and chatter bound $N_{0}$. The main result of this paper is that, for any family of $n \times n$ stability matrices $\mathcal{A} \triangleq\left\{A_{p}: p \in \mathcal{P}\right\}$, there is always an average dwell time $\tau_{D}$ such that (1) is uniformly exponentially stable over $\mathcal{S}_{\text {ave }}\left[\tau_{D}, N_{0}\right]$, for any chatter bound $N_{0}$. The next theorem formalizes this observation.

Theorem 2 Given a compact set of $n \times n$ matrices $\mathcal{A} \triangleq\left\{A_{p}: p \in \mathcal{P}\right\}$ and a positive constant $\lambda_{0}$ such that $A_{p}+\lambda_{0} I$ is asymptotically stable for each $p \in \mathcal{P}$, then, for any $\lambda \in\left[0, \lambda_{0}\right)$, there is a finite constant $\tau_{D}^{*}$ such that (1) is uniformly exponentially stable over $\mathcal{S}_{\text {ave }}\left[\tau_{D}, N_{0}\right]$ with stability margin $\lambda$, for any average dwell-time $\tau_{D} \geq \tau_{D}^{*}$ and any chatter bound $N_{0}>0$.

Before proceeding notice that, since each matrix $A_{p}+$ $\lambda_{0} I$ is asymptotically stable, there is a set of $n \times n$, symmetric, positive definite matrices $\mathcal{Q} \triangleq\left\{Q_{p}: p \in \mathcal{P}\right\}$ such that

$$
Q_{p}\left(A_{p}+\lambda_{0} I\right)+\left(A_{p}+\lambda_{0} I\right)^{\prime} Q_{p}=-I, \quad p \in \mathcal{P} .
$$

Moreover, since $\mathcal{A}$ is compact, $\mathcal{Q}$ is also compact. We therefore conclude that there is a family of Lyapunov functions

$$
\mathcal{V} \triangleq\left\{V_{p}: V_{p}(x) \triangleq x^{\prime} Q_{p} x, p \in \mathcal{P}\right\}
$$

for the time-invariant systems $\dot{z}=A_{p} z, p \in \mathcal{P}$, with the following properties:

(i) Each $V_{p}$ is continuous and decreases exponentially along solutions to the time-invariant system $\dot{z}=A_{p} z$. In particular,

$$
\frac{\partial V_{p}}{\partial x} A_{p} x \leq-2 \lambda_{0} V_{p}, \quad \forall x \in \mathbb{R}^{n}, p \in \mathcal{P} .
$$

(ii) There are functions $\alpha, \bar{\alpha}$ of $\operatorname{class}^{5} \mathcal{K}_{\infty}$ such that, for each $p \in \mathcal{P}$,

$$
\alpha(\|x\|) \leq V_{p}(x) \leq \bar{\alpha}(\|x\|), \quad \forall x \in \mathbb{R}^{n} .
$$

(iii) There is a positive constant $\mu$ such that

$$
V_{p}(x) \leq \mu V_{q}(x), \quad \forall x \in \mathbb{R}^{n}, p, q \in \mathcal{P} .
$$

Equation (6) is a straightforward consequence of (5) and equations (7) and (8) hold with

$$
\begin{gathered}
\alpha(s) \triangleq s^{2} \inf _{Q \in \mathcal{Q}} \sigma_{\min }[Q], \quad \bar{\alpha}(s) \triangleq s^{2} \sup _{\bar{Q} \in \mathcal{Q}} \sigma_{\max }[\bar{Q}], \\
\mu \triangleq \sup _{Q, \bar{Q} \in \mathcal{Q}} \frac{\sigma_{\max }[\bar{Q}]}{\sigma_{\min }[Q]}
\end{gathered}
$$

\footnotetext{
${ }^{5}$ We denote by $\mathcal{K}$ the set of all continuous functions $\alpha$ : $[0, \infty) \rightarrow[0, \infty)$ that are zero at zero, strictly increasing, and continuous, and by $\mathcal{K}_{\infty}$ the subset of $\mathcal{K}$ consisting of those functions that are unbounded.
}

where $\sigma_{\min }[Q]$ denotes the smallest singular value of $Q \in \mathcal{Q}$ and $\sigma_{\max }[\bar{Q}]$ the largest singular value of $\bar{Q} \in \mathcal{Q}$. In the above definitions the supremum and infimum are actually a maximum and minimum, respectively, due to the compactness of $\mathcal{Q}$. The existence of a family of Lyapunov functions with the above properties is the key technical result used in [19] to prove Theorem 2.

\section{Nonlinear Switched Systems}

In the sequel we extend the previous results to certain classes of nonlinear switched systems. To this effect consider a family $\mathcal{F} \triangleq\left\{F_{p}: p \in \mathcal{P}\right\}$ of nonlinear maps from $\mathbb{R}^{n}$ to itself such that $F_{p}(0)=0, p \in \mathcal{P}$. For each piecewise constant switching signal $\sigma$ we can then define the following switched non-linear system

$$
\dot{x}=F_{\sigma}(x) .
$$

The objective of this section is to derive conditions under which (9) is asymptotically stable in a uniform way over all slow-on-the-average switchings.

Given some family of piecewise constant switching signals $\mathcal{S}$, we say that (9) is uniformly asymptotically stable over $\mathcal{S}$ if there exists a function $\beta$ of $\operatorname{class}^{6} \mathcal{K} \mathcal{L}$ such that, for each $\sigma \in \mathcal{S}$,

$$
\|x(t)\| \leq \beta(\|x(\tau)\|, t-\tau), \quad \forall t \geq \tau \geq 0,
$$

along solutions to (9). When $\beta(s, t)$ is of the form $c e^{-\lambda t} s$ for some $c, \lambda>0$ we say that (1) is uniformly exponentially stable over $\mathcal{S}$ which, for the linear case, reduces to the definition in Section 2.

Clearly, for (9) to be uniformly asymptotically stable over any set $\mathcal{S}_{\text {ave }}\left[\tau_{D}, N_{0}\right], \tau_{D}, N_{0}>0$, the origin must be a globally, asymptotically stable equilibrium point of each time-invariant system $\dot{z}=F_{p}(z), p \in \mathcal{P}$. Here we actually demand more of the $F_{p}$ :

Assumption 3 There exist continuously differentiable functions $V_{p}: \mathbb{R}^{n} \rightarrow \mathbb{R}, p \in \mathcal{P}$, positive constants $\lambda_{0}$, $\mu$, and functions $\alpha, \bar{\alpha}$ of class $\mathcal{K}_{\infty}$ such that

$$
\begin{aligned}
\frac{\partial V_{p}}{\partial x} F_{p}(x) & \leq-2 \lambda_{0} V_{p}, \\
\alpha(\|x\|) & \leq V_{p}(x) \leq \bar{\alpha}(\|x\|), \\
V_{p}(x) & \leq \mu V_{q}(x),
\end{aligned}
$$

for each $x \in \mathbb{R}^{n}$ and $p, q \in \mathcal{P}$.

Equations (10)-(11) are the standard conditions for $V_{p}$ to be a Lyapunov function of $\dot{z}=F_{p}(z)$. It should be noted that, in light of $[20,21]$, the exponential decay suggested by (10) does not really introduce loss of

\footnotetext{
${ }^{6}$ We denote by $\mathcal{K} \mathcal{L}$ the set of continuous functions $\beta:[0, \infty) \times$ $[0, \infty) \rightarrow[0, \infty)$ which, for each fixed value of the second argument, are of class $\mathcal{K}$ when regarded as functions of the first argument, and that have $\lim _{\tau \rightarrow \infty} \beta(s, \tau)=0$ for each fixed $s \geq 0$.
} 
generality. As for (12), it may, in fact, reduce the set of systems to which the results in this section can be applied. Since (10)-(12) match exactly the equations (6)-(8) used to prove Theorem 2 , it is not surprising to discover that the following also holds true [19]:

Theorem 4 Given a set $\mathcal{F} \triangleq\left\{F_{p}: p \in \mathcal{P}\right\}$ of nonlinear maps from $\mathbb{R}^{n}$ to itself for which Assumption 3 holds, there is a finite constant $\tau_{D}^{*}$ such that (1) is uniformly asymptotically stable over $\mathcal{S}_{\text {ave }}\left[\tau_{D}, N_{0}\right]$, for any average dwell-time $\tau_{D} \geq \tau_{D}^{*}$ and any chatter bound $N_{0}>0$.

\section{Supervisory Control}

In this section we show how the previous results can be used in the context of supervisory control. We follow closely the formulation in $[1,5]$.

The problem addressed here is the set-point control of an imprecisely modeled process $\mathbb{P}$. In particular, we want to generate the control input $u$ to the process so as to drive its output $y$ to a constant reference $r$. The process has two other exogenous inputs that cannot be measured: a bounded measurement noise signal $\mathbf{n}$ and a bounded disturbance $\mathbf{d}$. For simplicity the signals $u, y, \mathbf{n}$, and $\mathbf{d}$ are scalar. $\mathbb{P}$ is assumed linear, time-invariant, with a stabilizable (through $u$ ) and detectable realization

$$
\dot{x}_{\mathbb{P}}=A_{\mathbb{P}} x+B_{\mathbb{P}} u+D_{\mathbb{P}} \mathbf{d}, \quad y=C_{\mathbb{P}} x+\mathbf{n},
$$

but precise values for $A_{\mathbb{P}}, B_{\mathbb{P}}, C_{\mathbb{P}}, D_{\mathbb{P}}$ are not known. It is known, however, that $\mathbb{P}$ 's transfer function $\tau$, from $u$ to $y$, belongs to a family of transfer functions of the form $\mathcal{N} \triangleq \bigcup_{p \in \mathcal{P}} \mathcal{N}_{p}$, where $p$ is an unknown parameter taking values in some parameter set $\mathcal{P}$ and each $\mathcal{N}_{p}$ denotes a family of transfer functions centered around a known, nominal transfer function $\nu_{p}$, e.g.,

$$
\mathcal{N}_{p} \triangleq\left\{(1+\delta) \nu_{p}:\|\delta\|_{\infty}<\epsilon\right\} .
$$

Here, $\epsilon$ denotes some small positive constant and $\delta$ a stable transfer function with $\mathcal{H}_{\infty}$-norm smaller than $\epsilon$. For simplicity, in the sequel we assume that $\epsilon=0$ and the set $\mathcal{P}$ is finite and equal to $\{1,2, \ldots, m\}$.

The solution proposed in $[1,5]$ to solve this problem is based on certainty equivalence and starts with the selection of a family of linear, time-invariant candidate controllers $\mathcal{C} \triangleq\left\{\kappa_{p}: p \in \mathcal{P}\right\}$. Each $\kappa_{p}$ would make the feedback closed-loop system in Figure 1 asymptotically stable if the process transfer function $\tau$ was known to belong to $\mathcal{N}_{p}$. To avoid pole-zero cancellations it is assumed that $\mathbb{P}$ does not have transmission zeros at the origin.

In case we knew to which set $\mathcal{N}_{p}$ the actual process transfer function $\tau$ belonged, stability of the closed loop could be achieved with a nonadaptive, linear,

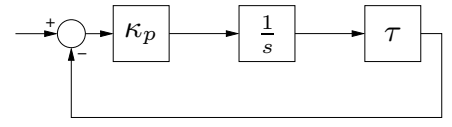

Figure 1: Feedback configuration.

time-invariant controller with transfer function equal to $\frac{1}{s} \kappa_{p}$. Since the process transfer function is not known in advance we build a "multi-controller" $\mathbb{C}$ that effectively allows switching between all the controller transfer functions in $\mathcal{C}$. If $\left\{\left(\bar{A}_{p}, \bar{B}_{p}, \bar{C}_{p}, \bar{D}_{p}\right): p \in \mathcal{P}\right\}$ is a family of $n$-dimensional, stabilizable and detectable realizations for the transfer functions in $\mathcal{C}$, the multicontroller $\mathbb{C}$ can be defined by

$$
\dot{x}_{\mathbb{C}}=\bar{A}_{\sigma} x_{\mathbb{C}}+\bar{B}_{\sigma} \mathbf{e}_{\mathbf{T}}, \quad v=\bar{C}_{\sigma} x_{\mathbb{C}}+\bar{D}_{\sigma} \mathbf{e}_{\mathbf{T}}, \quad \dot{u}=v,
$$

where $\mathbf{e}_{\mathbf{T}} \triangleq r-y$ and $\sigma:[0, \infty) \rightarrow \infty$ denotes a "switching signal" that, at each instant of time, determines which candidate controller is put into the feedback loop. The system that generates the switching signal $\sigma$ is called a supervisor. Here we are interested in estimator-based supervisors like the one in Figure 2. An estimator-based supervisor consists of three blocks:

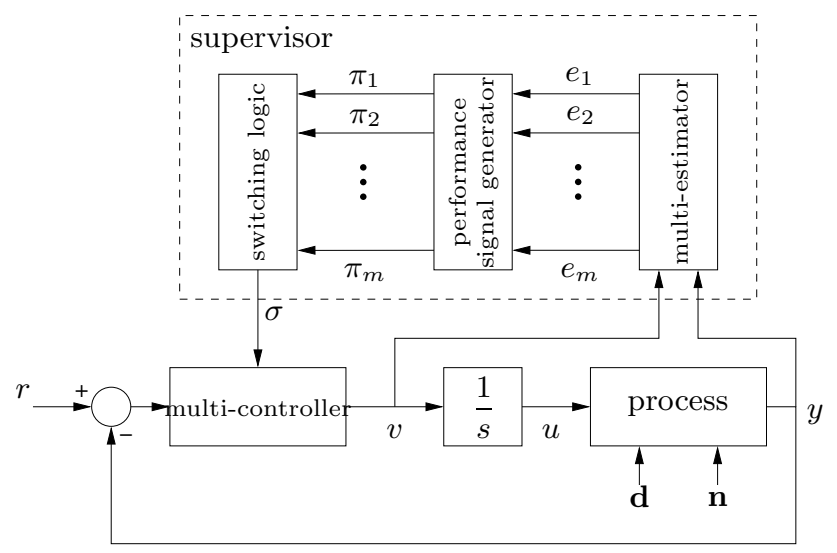

Figure 2: Supervisory control architecture.

a multi-estimator, a performance signal generator, and a switching logic.

The multi-estimator $\mathbb{E}$ is a linear, time-invariant system whose inputs are the outputs of the process and multi-controller and whose outputs are the output estimation errors $e_{p}, p \in \mathcal{P}$. Each $e_{p}$ is a signal that would converge to zero if the process transfer function $\tau$ was equal to the nominal transfer function $\nu_{p}$. The reader is referred to $[1,5]$ for the precise structure of $\mathbb{E}$. Denoting by $x$ the combined state of the multi-estimator and multi-controller (excluding the integrator), the evolution of $x$ is determined by

$$
\begin{aligned}
\dot{x} & =A_{\sigma} x+d_{\sigma} e_{\sigma}, \\
\mathbf{e}_{\mathbf{T}} & =c_{p^{*}} x+e_{p^{*}},
\end{aligned}
$$


where $A_{p}, d_{p}, c_{p}, p \in \mathcal{P}$ are appropriately defined matrices, and $p^{*}$ is the element of $\mathcal{P}$ for which $\tau \in \mathcal{N}_{p^{*}}$. Equation (15) is obtained from equation (23) in [1] with $l=\sigma$ and (16) is obtained from equation (26) in [1] with $l=p^{*}$. Moreover, there is a positive constant $\lambda_{0}$ for which each $\lambda_{0} I+A_{p}$ is asymptotically stable (cf. Remark 4 in [1]). From equation (28) in [1], one also concludes that $e_{p^{*}}$ is bounded and

$$
\begin{aligned}
\int_{0}^{t} e^{2 \lambda \tau} e_{p^{*}}(\tau)^{2} d \tau & \leq c_{n} e^{2 \lambda t}+c_{0}, & & t \geq 0, \\
\left\|e_{p^{*}}(t)\right\| & \leq d_{n}+d_{0} e^{-\lambda t}, & & t \geq 0,
\end{aligned}
$$

where $\lambda$ is any constant in $\left(0, \lambda_{0}\right), c_{0}, d_{0}$ are positive constants that depends only on initial conditions, and $c_{n}, d_{n}$ are positive constants that depends only on upper bounds on the norms of $\mathbf{n}$ and $\mathbf{d}$.

The performance signal generator $\mathbb{G}$ takes as inputs the output estimation errors $e_{p}, p \in \mathcal{P}$, and produces the performance signals $\pi_{p}, p \in \mathcal{P}$ defined by

$$
\dot{\tilde{\pi}}_{p}=-2 \lambda \tilde{\pi}_{p}+e_{p}^{2}, \quad \pi_{p}=\tilde{\pi}_{p}+\epsilon_{\pi}, \quad p \in \mathcal{P},
$$

with $\lambda \in\left(0, \lambda_{0}\right)$ and $\epsilon_{\pi}>0$ constant. $\mathbb{G}$ is initialized so that $\tilde{\pi}_{p}(0) \geq 0, p \in \mathcal{P}$.

The switching logic $\mathbb{S}$ generates the switching signal $\sigma$ based on the values of the performance signals $\pi_{p}$, $p \in \mathcal{P}$. The logic used here is called a scale-independent hysteresis switching logic and can be regarded as a hybrid dynamical system $\mathbb{S}_{\mathbb{H}}$ whose state and output are both $\sigma$. To specify $\mathbb{S}_{\mathbb{H}}$ it is necessary to first pick a positive number $h>0$ called a hysteresis constant. $\mathbb{S}_{\mathbb{H}}$ 's internal logic is then defined by the computer diagram shown in Figure 3 where, at each time $t$, $q \triangleq \arg \min _{p \in \mathcal{P}} \pi_{p}(t)$. The functioning of $\mathbb{S}_{\mathbb{H}}$ is roughly

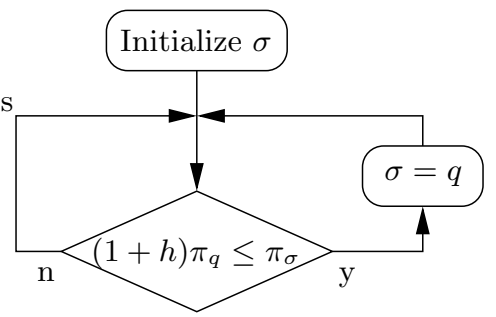

Figure 3: Computer Diagram of $\mathbb{S}_{\mathbb{S H}}$.

as follows. Suppose that at some time $t_{0}, \mathbb{S}_{\mathbb{H}}$ has just changed the value of $\sigma$ to $p$. The signal $\sigma$ is then held fixed at this value unless and until there is a time $t_{1}>t_{0}$ at which $(1+h) \pi_{q} \leq \pi_{p}$ for some $q \in \mathcal{P}$. If this occurs, $\sigma$ is set equal to $q$ and so on.

Suppose now that we define scaled performance signals $\bar{\pi}_{p} \triangleq \vartheta \pi_{p}$, with $\vartheta(t) \triangleq e^{2 \lambda t}, t \geq 0$. From (19) one concludes that, for each $t \geq t_{0} \geq 0$,

$$
\bar{\pi}_{p}(t)=\tilde{\pi}_{p}\left(t_{0}\right)+e^{2 \lambda t} \epsilon_{\pi}+\int_{t_{0}}^{t} e^{2 \lambda \tau} e_{p}(\tau)^{2} d \tau,
$$

and therefore each $\bar{\pi}_{p}$ is always monotone increasing and never smaller than $\epsilon_{\pi}$. By the Hysteresis Switching Theorem [17] we can then conclude that, for any $\ell \in \mathcal{P}$,

$$
N_{\sigma}\left(t, t_{0}\right) \leq 1+m+\frac{m}{\log (1+h)} \log \left(\frac{\bar{\pi}_{\ell}(t)}{\inf _{p \in \mathcal{P}} \bar{\pi}_{p}\left(t_{0}\right)}\right),
$$

for $0 \leq t_{0} \leq t<T$, where $N_{\sigma}\left(t_{0}, t\right)$ denotes the number of discontinuities of $\sigma$ on the interval $\left(t_{0}, t\right)$, and also

$$
\begin{aligned}
& \int_{t_{0}}^{t}\left(2 \lambda e^{2 \lambda \tau} \epsilon_{\pi}+e^{2 \lambda \tau} e_{\sigma}^{2}\right) d \tau \leq \\
& m\left((1+h) \bar{\pi}_{\ell}(t)-\inf _{p \in \mathcal{P}} \bar{\pi}_{p}\left(t_{0}\right)\right),
\end{aligned}
$$

for $0 \leq t_{0} \leq t<T$. Here we used the fact that $\frac{\mathrm{d}}{\mathrm{d} \tau}\left(\bar{\pi}_{\sigma(\tau)}(\tau)\right)=2 \lambda e^{2 \lambda \tau} \epsilon_{\pi}+e^{2 \lambda \tau} e_{\sigma}^{2}$, wherever the derivative exists. Now, from (17) and (20) we obtain $\bar{\pi}_{p^{*}}(t) \leq$ $e^{2 \lambda t}\left(\epsilon_{\pi}+c_{n}\right)+\bar{c}_{0}, t \geq 0$, where $\bar{c}_{0} \triangleq c_{0}+\tilde{\pi}_{p^{*}}(0)$. From this, (21)-(22) with $\ell \triangleq p^{*}$, and the fact that $\bar{\pi}_{p}\left(t_{0}\right) \geq e^{2 \lambda t_{0}} \epsilon_{\pi}, p \in \mathcal{P}$, we conclude that

$$
\begin{gathered}
N_{\sigma}\left(t, t_{0}\right) \leq 1+m+\frac{m \log \left(e^{2 \lambda\left(t-t_{0}\right)}\left(1+\frac{c_{n}}{\epsilon_{\pi}}\right)+\frac{\bar{c}_{0}}{\epsilon_{\pi}}\right)}{\log (1+h)}, \\
\int_{t_{0}}^{t} e^{2 \lambda \tau} e_{\sigma}^{2} d \tau \leq m\left((1+h)\left(e^{2 \lambda t}\left(\epsilon_{\pi}+c_{n}\right)+\bar{c}_{0}\right)\right. \\
\left.-e^{2 \lambda t_{0}} \epsilon_{\pi}\right)-\left(e^{2 \lambda t}-e^{2 \lambda t_{0}}\right) \epsilon_{\pi},
\end{gathered}
$$

for every $t \geq t_{0} \geq 0$. Since for $a, b>0, \log (a+b) \leq$ $\log (2 a)+\log (2 b)$, we also conclude that

$$
\begin{gathered}
N_{\sigma}\left(t, t_{0}\right) \leq N_{0}+\frac{t-t_{0}}{\bar{\tau}_{D}} \\
\int_{t_{0}}^{t} e^{2 \lambda \tau} e_{\sigma}^{2} d \tau \leq \bar{m} e^{2 \lambda t} c_{n}+\bar{m} \bar{c}_{0}-(m-1) e^{2 \lambda t_{0}} \epsilon_{\pi} \\
+(\bar{m}-1) e^{2 \lambda t} \epsilon_{\pi}
\end{gathered}
$$

with $\tau_{D} \triangleq \frac{\log (1+h)}{2 \lambda m}, \bar{m}=m(1+h)$, and

$$
N_{0} \triangleq 1+m+\frac{m}{\log (1+h)} \log \left(\frac{4 \bar{c}_{0}}{\epsilon_{\pi}}\left(1+\frac{c_{n}}{\epsilon_{\pi}}\right)\right) .
$$

Now, because of Lemma 1 and Theorem 2, there is a finite constant $\tau_{D}^{*}$ such that (15) has input-to-state $e^{\lambda t}$-weighted $\mathcal{L}_{2}$-to- $\mathcal{L}_{\infty}$ norm uniformly bounded over $\mathcal{S}_{\text {ave }}\left[\tau_{D}^{*}, N_{0}\right]$. If we then choose $\lambda$ and $h$ so that $\frac{\lambda}{\log (1+h)} \leq \gamma \triangleq \frac{1}{2 m \tau_{D}^{*}}$, we get $\tau_{D} \geq \tau_{D}^{*}$ and the output $\sigma$ of the switching logic is guaranteed to be in $\mathcal{S}_{\text {ave }}\left[\tau_{D}^{*}, N_{0}\right]$. From this and (24) one concludes that $x$ is bounded and, because of (14) and (16), $\mathbf{e}_{\mathbf{T}}$ and $v$ are also bounded. The boundedness of $u$ and the internal state of the process follows from the detectability of the cascade formed by the integrator in (14) and the process (13). The following can then be stated.

Theorem 5 There exists a positive constant $\gamma$ such that, whenever $\frac{\lambda}{\log (1+h)} \leq \gamma$, all signals remain bounded, for any bounded $\mathbf{n}$ and $\mathbf{d}$, and any initialization of $\mathbb{P}, \mathbb{E}, \mathbb{C}, \mathbb{G}, \mathbb{S}$, with $\tilde{\pi}_{p}(0) \geq 0, p \in \mathcal{P}$. 


\section{Conclusions}

We showed that switching among stable linear systems results in a stable system provided that switching is sufficiently slow on the average. In particular, it was shown that exponential stability is achieved when the number of switches in any finite interval grows linearly with the length of the interval, and the growth rate is sufficiently small. This was used to analyze a supervisory control algorithm using scale-independent hysteresis switching. The analysis took noise and disturbances into account and, to the best of our knowledge, is the first analysis of an hysteresis switching logic that does not rely on switching stopping. In a paper under preparation, the analysis presented here is extended to processes with unmodeled dynamics.

\section{Acknowledgments}

The authors would like to thank Daniel Liberzon for several discussions that lead to some of the results presented here.

\section{References}

[1] A. S. Morse, "Supervisory control of families of linear set-point controllers - part 1: exact matching," IEEE Trans. Automat. Contr., vol. 41, pp. 1413-1431, Oct. 1996.

[2] A. S. Morse, "Control using logic-based switching," in Trends in Control: A European Perspective (A. Isidori, ed.), pp. 69-113, London: Springer-Verlag, 1995.

[3] M. Chang and E. J. Davison, "Switching control of a family of plants," in Proc. of the 14th Amer. Contr. Conf., vol. 1, pp. 1015-1020, June 1995.

[4] K. S. Narendra and J. Balakrishnan, "Adaptive control using multiple models," IEEE Trans. Automat. Contr., vol. 42, pp. 171-187, Feb. 1997.

[5] A. S. Morse, "Supervisory control of families of linear set-point controllers - part 2: robustness," IEEE Trans. Automat. Contr., vol. 42, pp. 1500-1515, Nov. 1997.

[6] F. M. Pait and F. Kassab, Jr., "Parallel algorithms for adaptive control: Robust stability," in Control Using Logic-Based Switching (A. S. Morse, ed.), no. 222 in Lecture Notes in Control and Information Sciences, pp. 262-276, London: Springer-Verlag, 1997.

[7] J. Hockerman-Frommer, S. R. Kulkarni, and P. J. Ramadge, "Controller switching based on output prediction errors," IEEE Trans. Automat. Contr., vol. 43, pp. 596-607, May 1998.
[8] G. Chang, J. P. Hespanha, A. S. Morse, M. Netto, and R. Ortega, "Supervisory field-oriented control of induction motors with uncertain rotor resistance," in Proc. of the 1998 IFAC Workshop on Adaptive Control and Signal Processing, Glasgow, Scotland, Aug. 1998.

[9] J. P. Hespanha, Logic-Based Switching Algorithms in Control. PhD thesis, Yale University, New Haven, CT, 1998.

[10] S. Fujii, J. P. Hespanha, and A. S. Morse, "Supervisory control of families of noise suppressing controllers," in Proc. of the 37th Conf. on Decision and Contr., Dec. 1998.

[11] J. P. Hespanha and A. S. Morse, "Certainty equivalence implies detectability," Syst. 6 Contr. Lett., vol. 36, pp. 1-13, Jan. 1999.

[12] J. P. Hespanha, D. Liberzon, and A. S. Morse, "Towards the supervisory control of uncertain nonholonomic systems," in Proc. of the 1999 American Contr. Conf., June 1999.

[13] J. P. Hespanha and A. S. Morse, "Supervisory control of integral-input-to-state stabilizing controllers." To be presented at the 1999 European Control Conference., Aug. 1999.

[14] J. P. Hespanha and A. S. Morse, "Towards the supervisory control of families of nonlinear regulators," in Proc. of the 30th Annual Conf. on Information Sciences and Syst., vol. 2, pp. 765-768, Mar. 1996.

[15] R. H. Middleton, G. C. Goodwin, D. J. Hill, and D. Q. Mayne, "Design issues in adaptive control," IEEE Trans. Automat. Contr., vol. 33, pp. 50-58, Jan. 1988.

[16] A. S. Morse, D. Q. Mayne, and G. C. Goodwin, "Applications of hysteresis switching in parameter adaptive control," IEEE Trans. Automat. Contr., vol. 37, pp. 1343-1354, Sept. 1992.

[17] J. P. Hespanha and A. S. Morse, "Bounds on switching with scale-independent hysteresis." To be submitted for publication., Jan. 1999.

[18] S. R. Kulkarni and P. J. Ramadge, "Model and controller selection policies based on output prediction errors," IEEE Trans. Automat. Contr., vol. 41, pp. 1594-1604, Nov. 1996.

[19] J. P. Hespanha and A. S. Morse, "Stability of switched systems with average dwell-time," tech. rep., EE-Systems, University of Southern California / Lab. for Control Science \& Eng., Yale University, Jan. 1999.

[20] E. D. Sontag and Y. Wang, "New characterizations of input-to-state stability," IEEE Trans. Automat. Contr., vol. 41, pp. 1283-1294, Sept. 1996.

[21] L. Praly and Y. Wang, "Stabilization in spite of matched unmodeled dynamics and an equivalent definition of input-to-state stability," Math. Contr., Signals, Syst., vol. 9, pp. 1-33, 1996. 